\title{
False Covid-19 Test Results - Could We Do Things Better? A Personal Opinion
}

\author{
Suzanne Ekelund \\ Retired Principal Specialist, Clinical Biochemist, M. Sci. \\ Roedovre, Denmark \\ Email: suzanne.ekelund [AT] gmail.com
}

\begin{abstract}
This paper describes the problems with false covid-19 test results, both false positive and false negative results. The problems are related to the quality of tests, test sampling and the currently limited follow-up procedures. A test and follow-up strategy that could decrease the potential problems is suggested.
\end{abstract}

Keywords - covid-19, test strategy, positive predictive value, false positive results, false negative results

\section{INTRODUCTION}

During the covid-19 pandemic it seems as if the healthcare system has abandoned the important rule of interpreting all laboratory results in the clinical context. This important rule means that a single lab result cannot alone determine the diagnosis. An example could be that we find highly elevated pregnancy hormone levels in a male (which can happen). We should not then go to the patient and say: "Congratulations John. You are pregnant."

In many countries, including Denmark, everyone can get a covid-19 test, independently of whether they have symptoms or not, have been at risk of getting infected or work with vulnerable persons or patients. We have to ask ourselves if that is the most efficient and informative test strategy both for the patients and for the healthcare system and also whether it is the most economical test strategy.

No test is always giving the correct result. You sometimes get false negative results, that is negative results in patients who actually have the disease we are testing for, and you sometimes get false positive results, that is positive results in patients who do not have the disease we are testing for. That is why we have the rule of interpreting a test result in the clinical context.

In general, when you get a negative result in a patient who shows all signs of having the disease you have tested for, you either repeat the test or you do additional tests or assessments to clarify the situation. The same is the case if you get a positive result in a patient who shows no signs of having the disease you have tested for.

To estimate the potential problems with a false covid-19 result (negative as well as positive) we first need to look at the specifications of the covid-19 tests used and the number of persons receiving a false test result. Then we can look at potential countermeasures.

In April 2020 the Danish Health Authorities sent information to all general practitioners about the covid-19 test used for the throat swab tests [1]. According to this information the test used in Denmark is a PCR test and it has a sensitivity of $90-95 \%$ and a specificity of above $99 \%$ for patients with obvious respiratory symptoms. It is emphasized that the sensitivity and specificity for patients with only mild symptoms are not known and that the sampling technique has to be optimal to achieve the highest possible sensitivity.

Sensitivity is the fraction of persons with disease who will get a positive test. If sensitivity of a covid-19 test is 90-95 $\%$ it means that $5-10 \%$ of those who are infected will get a negative result. Reviews have found up to $29 \%$ of false negative results on PCR tests for covid-19 [2]. In Denmark you are told when you have your sample taken that you can check the result within three days on your personal page on the national health care digital platform. If your test was negative you get either the result "NEGATIVE" or "NOT DETECTED". Below it is explained that "NEGATIVE" or "NOT DETECTED" means that you are not infected with covid-19. There is no guidance to what you should do if have obvious symptoms of covid-19.

A patient who gets a negative result will trust the result and not stay in quarantine and thus risk infecting more persons. Up to $29 \%$ of all the infected persons cold be in this situation without knowing it [2].

Specificity is the fraction of patients without the disease tested for who will get a negative result. If the specificity of the covid-19 test is above $99 \%$ it means that less than $1 \%$ of all without disease will get a false positive result. As 
mentioned above, in Denmark you have to check the test result on your personal page on the national health care digital platform. If your test was positive you get the result "POSITIVE" or "DETECTED". Below it is explained that "POSITIVE" or "DETECTED" means that you are infected with covid-19. In additions you get a letter in your official ebox which is used when the authorities send you mails. In the letter it is explained how you should isolate yourself from others etc. The authorities also try to call the infected persons by phone to trace the infection chains. However, this part is in many cases not successful.

Now how many get a false positive result? To estimate that we need to know the number of persons being tested and how many of these did have disease. Then we can calculate the so-called positive predictive value, which tells us the percentage of all with a positive test who actually have the disease. $100 \%$ minus the positive predictive value is the fraction of tested persons who get a false positive result. The positive predictive value depends on the sensitivity and specificity of the test. However, the prevalence, i.e. the fraction of persons with disease in the population tested, also has a strong impact on the value.

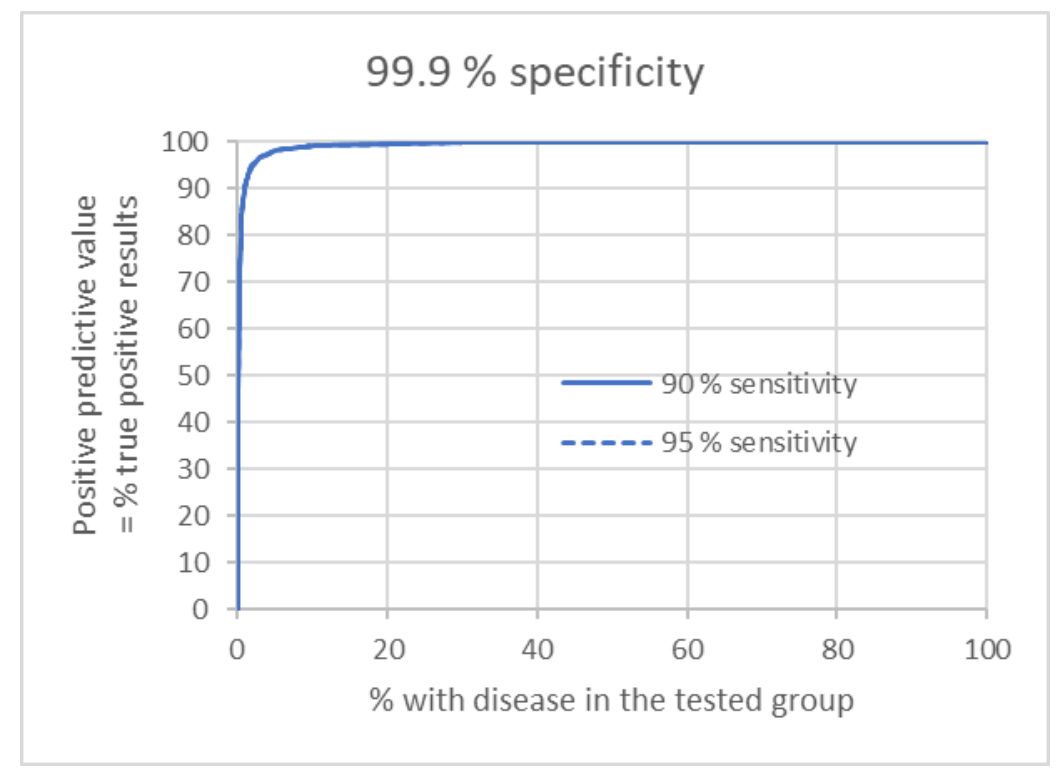

Figure 1: Positive predictive value for a test with $99.9 \%$ specificity. Calculated for both $90 \%$ sensitivity and $95 \%$ sensitivity.

As can be seen in Figure 1, the positive predictive value (PPV) increases with increasing prevalence of disease. To understand why that is, consider two populations:

- No one in the population has the disease (prevalence $=0 \%$ ). When there are only persons without disease in a population, every positive result is a false-positive result and the PPV is $0 \%$.

- $\quad$ Everyone in a population has the disease (prevalence $=100 \%$ ). When there are only persons with disease in a population, every positive result is a true-positive result and the PPV is $100 \%$.

The form of the curve depends on the sensitivity and the specificity of the test. As can be seen here there is not a clear visible difference between tests having $90 \%$ sensitivity vs. $95 \%$ sensitivity as long as they both have a specificity of 99.9 $\%$. 


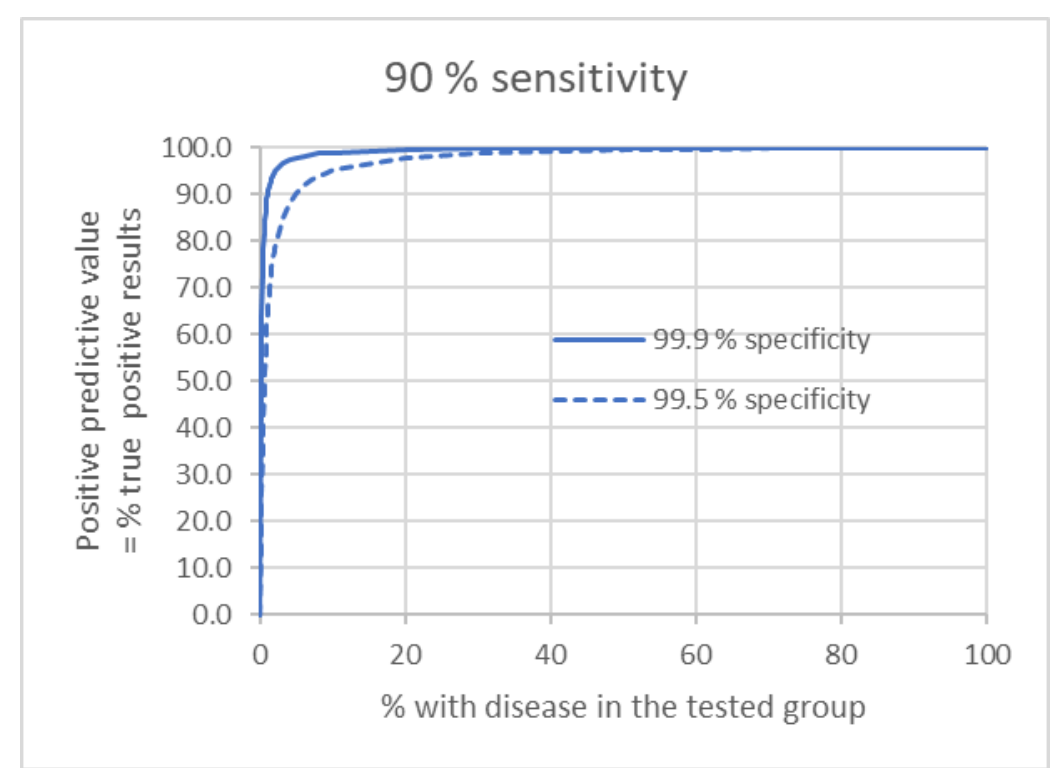

Figure 2: Positive predictive value for a test with $90 \%$ sensitivity. Calculated for both $99.9 \%$ specificity and $99.5 \%$ specificity.

As can be seen in Figure 2, the PPV differs in the low end (low fraction of persons with the disease) when the specificity differs between $99.9 \%$ vs. $99.5 \%$.

According to the Danish Health Authorities the fraction of persons tested positive in Denmark is around $1 \%$ of all persons tested [3]. Therefor we should have a closer look at the PPV in the low end.

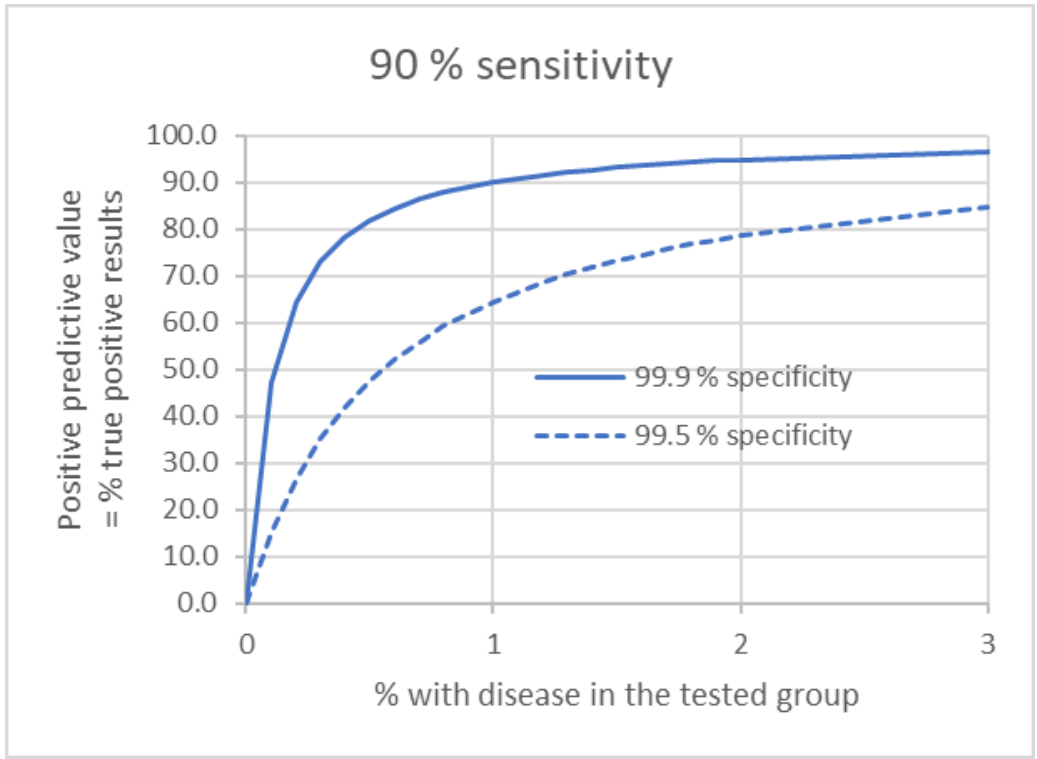

Figure 3: Positive predictive value for a test with $90 \%$ sensitivity. Calculated for both $99.9 \%$ specificity and $99.5 \%$ specificity. The low end of the curve is shown.

Figure 3 shows that with a $99.5 \%$ specificity about $66 \%$, that is two out of three of the positive results were true positives, while one third of the positive results were false positives. Even if the specificity was as high as $99.9 \%$ about one in ten of the positive results was a false positive. These high false positive rates are in agreement with what Wernike et al. [4] found. They found specificities down to $97.56 \%$ and they found that the problem of what we in this case would call a low specificity may be related to the so-called primers in certain batches of reagents, also in commercial kits.

Many would think that it is not important because if you get false positive result you do not have the disease and you do not infect anyone. However, it means that many people think that they have had covid-19 and they might fear getting some of the very problematic late onset complications described in previously covid-19 infected patients. These 
complications are not only linked to the lungs but also to other organs, i. e. the heart [5] and to thrombosis [6] and the number of complications increase as we gain more knowledge about the disease.

A change in test strategy and a follow-up procedure based on preset criteria could decrease the problems.

\section{SUGGESTED TEST STRATEGY}

Covid-19 infected patients do form antibodies. A study has shown that a week after onset of disease less than $40 \%$ of the patients had created antibodies but after 15 days, they had all created antibodies [7]. Unfortunately, it has also been shown that some patients do not have antibodies anymore a few months after disease [8]. Based on this you could create a better test strategy instead of the current "test, test, test".

Criterion for testing (one of the following):

- Symptoms of covid-19

- Exposure to covid-19

- Staff working with vulnerable persons or with covid-19 patients

Follow-up on positive test result:

- All without exemption should go into a 14-day quarantine

- Persons who do not have symptoms or non-obvious symptoms are offered an antibody test about two weeks later to assess whether their result was true positive or false positive

Follow-up on negative test result:

- Persons where there is a strong suspicion on infection should have the throat swab repeated and offered an antibody test about two weeks later

- $\quad$ Others - no follow-up

\section{CONCLUSION}

A more restrictive test strategy as the one suggested could increase the fraction of people with disease among the tested persons. This will increase the positive predictive value and decrease the number of persons getting a false positive result.

Follow-up based on preset criteria for both persons with positive and with negative results will give us a better knowledge of how many are actually infected and it will give the persons more certainty about their true result.

Finally, such a test strategy would be more aligned with good medical practice.

\section{REFERENCES}

[1] Sundhedsstyrelsen: Information om PCR test for COVID-19 til almen praksis. April 14,2020 (https://www.sst.dk//media/Udgivelser/2020/Corona/IRF-Almen-praksis/Kommunikation-til-almen-praksis test-afCOVID.ashx?la=da\&hash=A28AC860410928AAD8864F689B6A05C9E56F3A87)

[2] Keller J, Liyanage SE, Hingorani M, Hingorani A. Probability of encountering Covid-19 patients based on prevalence and testing during resumption of ophthalmology services. Eye (Lond) 2020;16:1-2. doi: 10.1038/s41433-020-1089-4. Online ahead of print. (https://www.ncbi.nlm.nih.gov/pmc/articles/PMC7364291/)

[3] Sundhedsstyrelsen. Tal og overvågning: COVID-19 (https://www.sst.dk/da/corona/tal-og-overvaagning)

[4] Wernike K, Keller M, Conraths FJ, Mettenleiter TC, Groschup MH, Beer M. Pitfalls in SARS-CoV-2 PCR diagnostics. Transbound Emerg Dis 2020;14:10.1111/tbed.13684. doi: 10.1111/tbed.13684. Online ahead of print. (https://www.ncbi.nlm.nih.gov/pmc/articles/PMC7323359/)

[5] Basso C, Leone O, Rizzo S, De Gaspari M, van der Wal AC, Aubry MC, Bois MC, Lin PT, Maleszewski JJ, Stone JR. Pathological features of COVID-19-associated myocardial injury: a multicentre cardiovascular pathology study.Eur Heart J 2020 Sep 24:ehaa664. doi: 10.1093/eurheartj/ehaa664. Online ahead of print.

[6] Wichmann D, Sperhake JP, Lütgehetmann M, Steurer S, Edler C, Heinemann A, Heinrich F, Mushumba H, Kniep I, Schröder AS, Burdelski C, de Heer G, Nierhaus A, Frings D, Pfefferle S, Becker H, Bredereke-Wiedling H, de Weerth A, Paschen HR, Sheikhzadeh-Eggers S, Stang A, Schmiedel S, Bokemeyer C, Addo MM, Aepfelbacher M, Püschel K, Kluge S. Autopsy Findings and Venous Thromboembolism in Patients With COVID-19: A Prospective Cohort Study. Ann Intern Med 2020 Aug 18;173(4):268-277. doi: 10.7326/M20-2003. Epub 2020 May 6. 
[7] Jacofsky D, Jacofski EM, Jacofski M. Understanding Antibody Testing for COVID-19. J Arthroplasty 2020; 35(7): S74-S81. (https://www.ncbi.nlm.nih.gov/pmc/articles/PMC7184973/)

[8] Liu A, Wang W, Zhao X, Yang D, Lu M, Lv Y. Disappearance of antibodies to SARS-CoV-2 in a -COVID-19 patient after recovery. Clin Microbiol Infect 2020; Epub ahead of print. (https://www.ncbi.nlm.nih.gov/pmc/articles/PMC7346807/) 